\title{
Alergia de Contacto a Metais num Período de 20 Anos no Centro de Portugal: Implicações das Directivas Europeias
}

\author{
Allergic Contact Dermatitis to Metals over a 20-Year Period in the Centre of \\ Portugal: Evaluation of the Effects of the European Directives
}

Vera TEIXEIRA ${ }^{1}$, Inês COUTINHO ${ }^{1}$, Margarida GONÇALO ${ }^{1}$

Acta Med Port 2014 May-Jun;27(3):295-303

\section{RESUMO}

Introdução: Os metais são causa frequente de dermatite de contacto alérgica. Desde a introdução da Directiva Comunitária do Níquel (1994/27/CE; 2004/96/CE) e, mais recentemente, da Directiva do Cimento (2003/53/CE), nos países nórdicos tem havido uma diminuição da sensibilização aos metais. A aplicabilidade destas medidas e o seu impacto permanece por avaliar em Portugal.

Material e Métodos: Foi realizado um estudo retrospectivo (1992-2011) na Consulta de Alergologia Cutânea com o principal objectivo de analisar a variação dos padrões de sensibilização aos metais (níquel [Ni], cobalto [Co] e crómio [Cr]) ao longo de 20 anos, em particular no que se refere ao género, grupo etário e relação com a actividade profissional.

Resultados: Do total de 5250 doentes estudados, em 1626 (31\%) observámos pelo menos um teste epicutâneo positivo a um metal $(26,5 \%$ ao $\mathrm{Ni} ; 10,0 \%$ ao Co e $7,0 \%$ ao $\mathrm{Cr}$ ). A prevalência de sensibilização ao Ni era significativamente maior nas mulheres $(34,4 \%$ versus $8,9 \%$ ) e ao $\mathrm{Cr}$ nos homens (11,5\% versus $5,0 \%$ ). Não houve diminuição significativa da sensibilização ao Ni ao longo dos 20 anos, mas o contributo da faixa etária dos 16-30 anos no total de mulheres sensíveis a este metal decresceu de forma significativamente estatística ao longo dos anos $(p<0,001)$. A sensibilização ao $\mathrm{Cr}$ decresceu sobretudo nos homens $(r=-0,535)$, muito particularmente nos casos relacionados com a construção civil $(r=-0,639)$, enquanto a reatividade ao Cr relacionado com o uso de calçado se manteve estável.

Discussão: A manutenção dos elevados níveis de sensibilização ao níquel denota ainda uma fraca implementação das Directivas comunitárias mas a redução da percentagem de mulheres jovens poderá significar já algum efeito positivo nesta faixa etária. Ao contrário, a Directiva referente à redução do $\mathrm{Cr}$ no cimento parece estar a ter os seus efeitos positivos. Há, contudo, necessidade de interferir com o conteúdo em $\mathrm{Cr}$ no couro natural utilizado no calçado.

Conclusões: A regulamentação da implementação de medidas interventivas relacionadas quer com o fabrico e comércio de adornos quer a nível profissional permitirá uma melhor protecção da população da alergia aos metais.

Palavras-chave: Dermatite de Contacto Alérgica; Metais; Níquel; Cobalto; Crómio; Testes Epicutâneos; Portugal; União Europeia.

\section{ABSTRACT}

Introduction: Metals are a common cause of allergic contact dermatitis. After the introduction of the EU Nickel Directive (1994/27/CE; 2004/96/EC) and, more recently, the Cement Directive (2003/53/EC) there has been a significant decrease in sensitization to metals mainly in the Nordic countries. The applicability of these directives and their impact in the Portuguese population is unknown.

Material and Methods: A retrospective study (1992-2011) was carried out in our patch test clinic to assess the temporal trend of metal sensitization (nickel [Ni], cobalt [Co] and chromium [Cr]) along the last 20 years, particularly considering age, sex and its relation with occupational activity.

Results: Out of 5250 consecutively patch-tested patients, $1626(31 \%)$ were reactive to at least one metal $(26.5 \%$ to $\mathrm{Ni}$; $10.0 \%$ to $\mathrm{Co}$ and $7.9 \%$ to $\mathrm{Cr}$ ). Women had a higher prevalence of sensitization to $\mathrm{Ni}(34.4 \%$ vs $8.9 \%)$ whereas men were more reactive to $\mathrm{Cr}$ $(11.5 \%$ vs $5.0 \%)$. Nickel sensitization did not decrease significantly over the years, although in recent years among women sensitized to nickel the percentage of younger patients $(16-30$ years-old) is significantly lower $(p<0.001)$. Chromium sensitization significantly decreased, particularly in men $(r=-0.535)$, and mainly in the construction workers $(r=-0.639)$. Chromium reactivity associated with the shoe dermatitis has remained stable.

Discussion: We emphasize the higher and stable percentage of nickel sensitized individuals suggesting, so far, a low impact from the EU Ni directive, although a decreasing percentage in the the younger group among Ni sensitized women may suggest a beneficial effect is becoming evident is this age group. On the contrary, the impact of the directive regarding the modification of $\mathrm{Cr}$ in cement seems to be effective. There is now a need to regulate chromium content in leather products, namely in shoes.

Conclusions: The regulation of interventional measures related either to the manufacture and trade of adornments or professional use will better protect the population of allergy to metals.

Keywords: Dermatitis, Allergic Contact; Chromium; Cobalt; European Union; Metals; Nickel; Patch Tests; Portugal.

\section{INTRODUÇÃo}

Os metais são causa frequente de alergia de contacto e dermatite de contacto alérgica (DCA). Durante o século $X X$, nos países industrializados, a sensibilização ao níquel (Ni) tornou-se a causa mais frequente de DCA nas muIheres. $^{1,2}$ Tal deve-se a uma maior exposição através dos adornos metálicos em contacto directo e prolongado com a pele, como por exemplo a bijuteria e partes metálicas do vestuário. $\mathrm{O} \mathrm{Ni}$ está ainda presente em inúmeros objectos metálicos usados no dia-a-dia (chaves, relógios, isqueiros, tesouras, dedais, pinças, armações de óculos, telemóveis, moedas de 1 e 2 euros, etc.), próteses dentárias e ortopédicas. ${ }^{3-8}$ Nos indivíduos sensibilizados, a exposição cutânea ao $\mathrm{Ni}$ em concentrações superiores ao limite é capaz de desencadear a DCA, o contacto com próteses metálicas or-

1. Serviço de Dermatologia. Centro Hospitalar e Universitário de Coimbra. Coimbra. Portugal.

Recebido: 11 de Junho de 2013 - Aceite: 19 de Julho de 2013 | Copyright @ Ordem dos Médicos 2014 
topédicas ou dentárias poderá desencadear reações locais de intolerância e a sua absorção pode causar dermatite de contacto sistémica. ${ }^{9}$

A Directiva Comunitária do Níquel (1994/27/EC), implementada em Junho de 1994, e com efeito em Portugal a partir de Outubro de 2000, estabelece que o $\mathrm{Ni}$ ou os seus compostos só poderão utilizar-se: i) em conjuntos de hastes inseridas, a título temporário ou não, em orelhas furadas e noutras partes perfuradas (brincos, piercings, etc.) em que a libertação de níquel seja inferior a $0,5 \mu \mathrm{g} / \mathrm{cm}^{2}$ por semana e, ii) em produtos destinados a entrar em contacto directo e prolongado com a pele se a taxa de libertação de níquel destes objectos for inferior a $0,5 \mu \mathrm{g} / \mathrm{cm}^{2}$ por semana ${ }^{10}$. Em 2004, reconsiderando que os limites previamente impostos eram insuficientes, a Directiva foi alterada (2004/96/EC), restringindo a colocação no mercado e a utilização de conjuntos de piercing com libertação de $\mathrm{Ni}$ em contacto com a pele inferior a $0,2 \mu \mathrm{g} / \mathrm{cm}^{2}$ por semana, modificação que entrou em vigor em Portugal em Dezembro de 2005. ${ }^{11}$ Mais recentemente, como se verificaram vários casos de DCA ao níquel por contacto com porções metálicas de telemóveis, o conteúdo em $\mathrm{Ni}$ destes foi também abrangido por regulamentação Comunitária.

O cobalto (Co) partilha as fontes de exposição com o $\mathrm{Ni}$ e o crómio $(\mathrm{Cr})$, nomeadamente a nível profissional na construção civil com a manipulação do cimento, produtos de cabedal, porcelana e tintas com pigmento azul/azul esverdeado e amarelos para cerâmica, vidro, esmalte e tinta de impressão. É ainda um importante alérgeno presente nos adornos metálicos (bijuteria) e partes metálicas do vestuário, próteses dentárias e ortopédicas, tatuagens, cosméticos e tintas capilares (cor castanho claro). Deste modo, frequentemente a alergia ao Co acompanha a alergia ao $\mathrm{Cr}$ e/ou $\mathrm{Ni}$, por sensibilização concomitante. . $^{3-5}$

O crómio é um alérgeno importante do ponto de vista profissional, nomeadamente na construção civil com o manuseio do cimento, contacto com produtos de cabedal e na indústria de curtimento, como pigmento em tintas anti-corrosivas. $\mathrm{Na}$ exposição não profissional, salienta-se o contacto com produtos de cabedal (calçado, correias de relógio, luvas, cinto, etc.), manipulação de produtos de limpeza (lixívias amarelas), maquilhagem e próteses metálicas ortopédicas. ${ }^{3}$ A Directiva do cimento (2003/53/CE), implementada em Junho de 2003 na Europa e em Portugal em Março de 2005, estabelece que o cimento, quando hidratado, não pode ter uma quantidade de $\mathrm{Cr}$ VI solúvel superior a $0,0002 \%$ (duas partes por milhão [ppm]) do seu peso seco total. ${ }^{12}$

Estas medidas preventivas têm como objectivo proteger os consumidores europeus da alergia ao $\mathrm{Ni}$ e $\mathrm{Cr}$ que, como referimos, pode ter implicações além da DCA. A evidência científica mostra que com estes limites, poderá ocorrer menor sensibilização, e, eventualmente, evitar DCA em indivíduos previamente sensibilizados. ${ }^{13}$ Desde a introdução destas Directivas comunitárias, nos países nórdicos tem havido uma diminuição da sensibilização aos metais. ${ }^{14,15}$ A aplicabilidade destas medidas e o seu grau de impacto permanece por avaliar em Portugal.

\section{OBJECTIVOS}

Com o objectivo de avaliar um eventual impacto destas medidas na população do centro de Portugal, o presente estudo investigou a prevalência de alergia ao $\mathrm{Ni}$, Co e $\mathrm{Cr}$ numa população de doentes submetidos a testes epicutâneos (TE), e sua variação durante um período de 20 anos.

\section{MATERIAL E MÉTODOS}

Foram incluídos no estudo todos os doentes com suspeita de DCA ou outra reacçâo cutânea de hipersensibilidade submetidos a TE entre Janeiro de 1992 e Dezembro de 2011, no Centro Hospitalar e Universitário de Coimbra. A sensibilização aos metais foi definida como reação positiva a pelo menos um dos três metais testados na série básica de alérgenos ( $\mathrm{Ni}, \mathrm{Co}, \mathrm{Cr})$.

A relevância clínica foi categorizada como i) relevância actual - o TE revelou o alérgeno que causa a dermatite, ii) relevância passada - a reação não tem relevância para a dermatite actual, mas justifica situações prévias de DCA iii) não relevante - não foi encontrada explicação para o TE positivo.

Foi avaliada a variação da reactividade aos metais no seu conjunto, e de forma isolada a cada um dos três metais, em grupos de cinco anos ao longo dos 20 anos do estudo e caracterizados os padrões de evolução em função do género, idade e a sua relevância para a atividade profissional.

\section{Testes epicutâneos}

Os alérgenos metálicos testados na série básica do Grupo Português de estudo das Dermatites de Contacto incluíram o sulfato de níquel $5 \%$ vas., cloreto de cobalto $1 \%$ vas. e dicromato de potássio $0,5 \%$ vas. (Chemotechnique Diagnostics $^{\mathrm{TM}}$, Vellinge, Sweden). Foram aplicados no tronco posterior em Finn Chambers on Scanpor Tape (Epitest Ld, Amirall) ou, mais raramente, em IQ chambers (Chemotechnique Diagnostics ${ }^{\mathrm{TM}}$, Vellinge, Sweden) e removidos ao fim de 48h. As leituras foram realizadas em D2 e D4, D3 e D4 ou, eventualmente, D7, segundo as recomendações do International Contact Dermatitis Research Group ${ }^{16}$. Foram consideradas positivas as reações 1+ (eritema e pápulas ou infiltração) ou mais intensas (2+ e $3+)$.

\section{Análise estatística}

A análise estatística foi realizada com o SPSS (Software Package for Statistical Science, versão 18.0, Chicago, IL). Foram aplicados testes paramétricos e não-paramétricos para analisar os padrões de evolução da sensibilização aos metais. $O$ valor $p$ igual ou inferior a 0,05 foi considerado significativo.

\section{RESULTADOS}

Ao longo dos 20 aos foram estudados 5250 indivíduos ( 3636 do sexo feminino $-69,3 \%$ e 1614 do sexo masculino - 30,7\%), com idade média de 37 anos, $23,1 \%$ 
Tabela 1 - Dados demográficos e resultados dos testes epicutâneos da população estudada.

TE: Testes epicutâneos; a variável 'Reactividade relevante' inclui relevância actual e relevância passada; TP/TNP: testes paramétricos/ testes não-paramétricos, conforme indicado; quando $p<0,05$, foi aplicada a correlação de Pearson ( $r$ ), nos testes paramétricos e a correlação de Spearman (ro), nos testes não-paramétricos, respectivamente. Nota: Para efeitos de apresentação dos dados, foram agrupados $5 / 5$ anos, mas a análise estatística foi efectuada com os dados dos 20 anos.

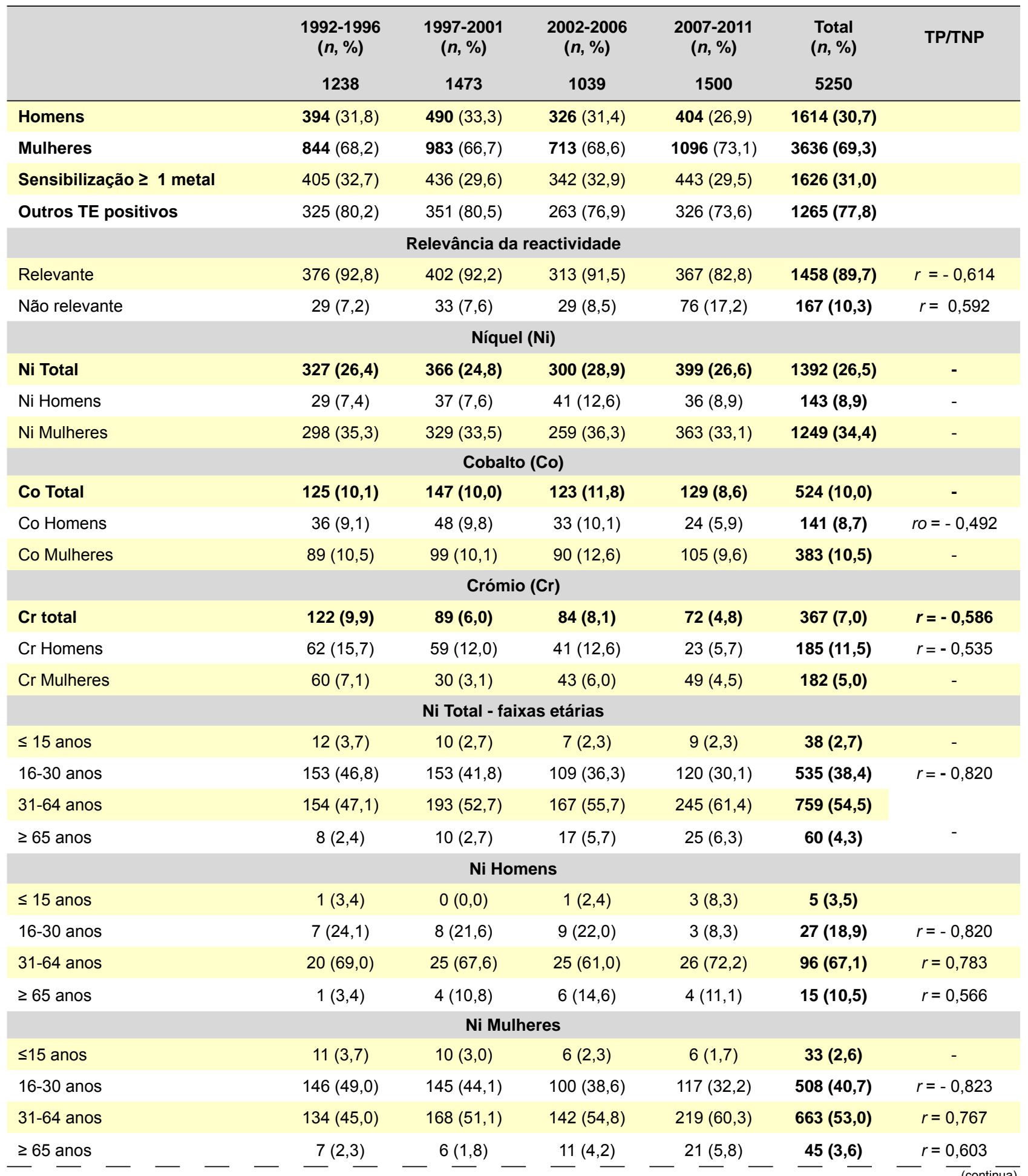

com doença ocupacional, 35,9\% com dermatite das mãos, $6,5 \%$ com úlcera de perna ou insuficiência venosa crónica dos membros inferiores, $20,6 \%$ com envolvimento facial e $24,1 \%$ com história pessoal de atopia.

Do total de 5250 doentes, 1626 (31\%) apresentaram pelo menos um TE positivo a um metal: $1392(26,5 \%)$ ao
Ni, $524(10,0 \%)$ ao Co e $367(7,0 \%)$ ao Cr (Tabela 1). Sensibilização ao Ni foi observada em $34,4 \%$ das mulheres vs $8,9 \%$ dos homens $(p<0,05)$, nestes sobretudo no grupo etário dos 30-64 anos. A reactividade ao Co ocorreu em $10,5 \%$ dos indivíduos do sexo feminino e $8,7 \%$, do sexo masculino. A reatividade ao $\mathrm{Cr}$ foi mais frequente no sexo 
Tabela 1 - Dados demográficos e resultados dos testes epicutâneos da população estudada. (continuação)

TE: Testes epicutâneos; a variável 'Reactividade relevante' inclui relevância actual e relevância passada; TP/TNP: testes paramétricos/ testes não-paramétricos, conforme indicado; quando $p<0,05$, foi aplicada a correlação de Pearson ( $r$ ), nos testes paramétricos e a correlação de Spearman (ro), nos testes não-paramétricos, respectivamente. Nota: Para efeitos de apresentação dos dados, foram agrupados $5 / 5$ anos, mas a análise estatística foi efectuada com os dados dos 20 anos.

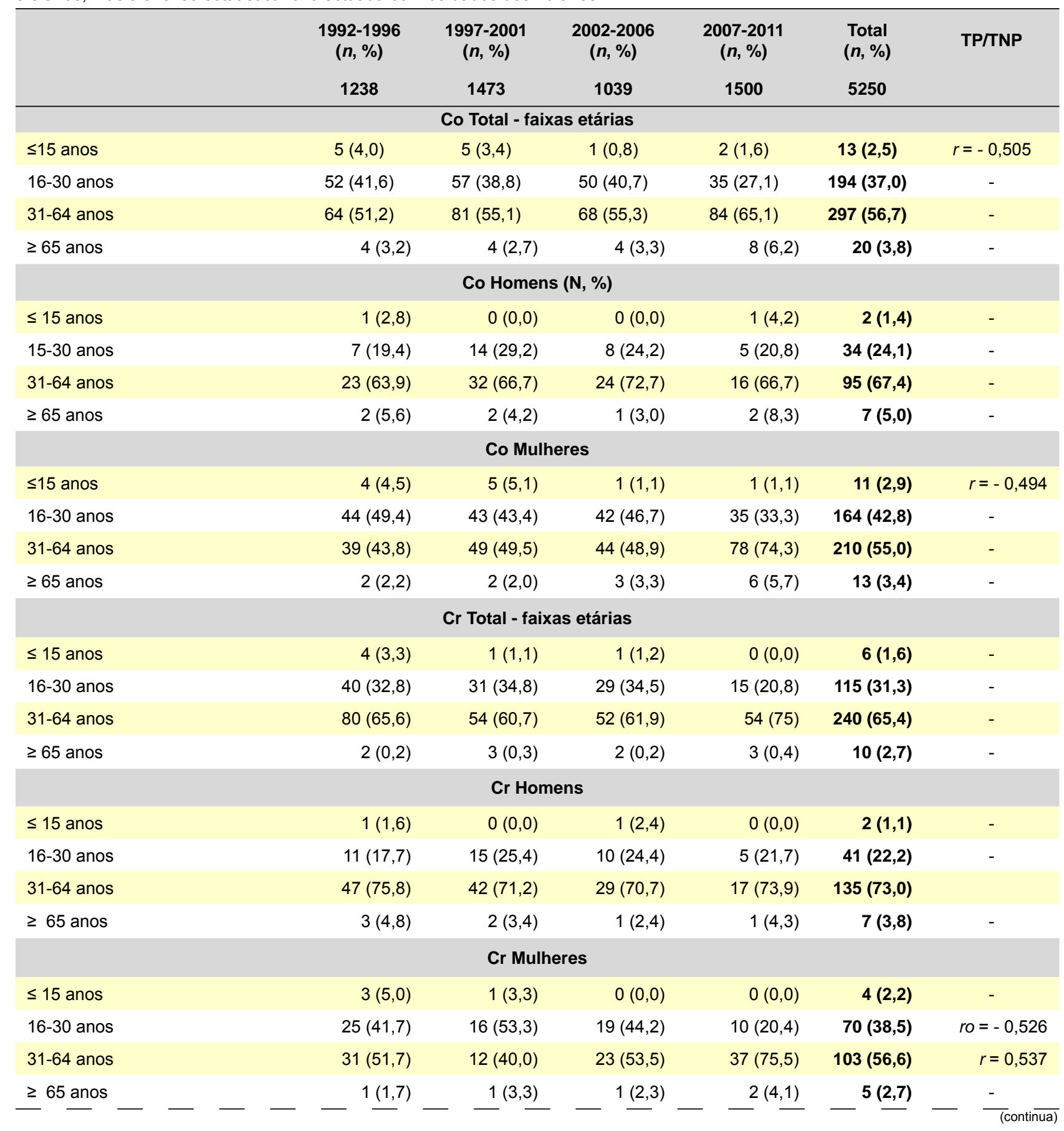

masculino $(11,5 \%$ vs $5,0 \%, p<0,05)$ e em idades mais tardias sobretudo no sexo masculino, em que o grupo acima dos 30 anos correspondia a $76,8 \%$ dos indivíduos reactivos ao $\mathrm{Cr}$ (Tabela 1).

Testes epicutâneos positivos a três metais ocorreram em 120 (2,3\%) indivíduos. A associação de TE positivos ao $\mathrm{Ni}$ e Co ocorreu em 389 (7,4\%), sobretudo no sexo feminino $(9,2 \%)$, e associação ao $\mathrm{Cr}$ e Co foi observada em 211 $(4,0 \%)$, sobretudo no sexo masculino $(6,6 \%)$. Foi mais rara a associação entre o Ni e $\mathrm{Cr}(n=178,3,4 \%)$ (Fig. 1).

Como sugerido pela análise do padrão temporal de variação da sensibilização aos metais (Fig. 2) e confirmado pela análise estatística, não houve diminuição significativa na sensibilização aos metais em geral $(p>0,05)$. Também, nestes 20 anos, a sensibilização ao Ni considerada de forma isolada, manteve-se estável, não sendo distinto o padrão evolutivo com os sexos. Quando analisado em função da idade verificou-se que a faixa etária dos 16-30 
Tabela 1 - Dados demográficos e resultados dos testes epicutâneos da população estudada. (secção final)

TE: Testes epicutâneos; a variável 'Reactividade relevante' inclui relevância actual e relevância passada; TP/TNP: testes paramétricos/ testes não-paramétricos, conforme indicado; quando $p<0,05$, foi aplicada a correlação de Pearson ( $r$ ), nos testes paramétricos e a correlação de Spearman (ro), nos testes não-paramétricos, respectivamente. Nota: Para efeitos de apresentação dos dados, foram agrupados $5 / 5$ anos, mas a análise estatística foi efectuada com os dados dos 20 anos.

\begin{tabular}{|c|c|c|c|c|c|c|}
\hline & $\begin{array}{c}1992-1996 \\
(n, \%) \\
1238 \\
\end{array}$ & $\begin{array}{c}1997-2001 \\
(n, \%) \\
1473 \\
\end{array}$ & $\begin{array}{c}2002-2006 \\
(n, \%) \\
1039 \\
\end{array}$ & $\begin{array}{c}2007-2011 \\
(n, \%) \\
1500 \\
\end{array}$ & $\begin{array}{c}\begin{array}{c}\text { Total } \\
(n, \%)\end{array} \\
5250 \\
\end{array}$ & TPITNP \\
\hline \multicolumn{7}{|c|}{ Co-sensibilização } \\
\hline $\mathrm{Ni}+$ Co Total & $91(7,4)$ & $103(7,0)$ & $96(9,2)$ & $99(6,6)$ & $389(7,4)$ & - \\
\hline $\mathrm{Ni}+$ Co Homens & $11(2,8)$ & $14(2,9)$ & $17(5,2)$ & $11(2,7)$ & $53(3,3)$ & - \\
\hline $\mathrm{Ni}+$ Co Mulheres & $80(9,5)$ & $89(9,1)$ & $79(11,1)$ & $88(8,0)$ & $336(9,2)$ & - \\
\hline $\mathrm{Ni}+\mathrm{Cr}$ Total & $50(4,0)$ & $30(2,0)$ & $50(4,8)$ & $48(3,2)$ & $178(3,4)$ & - \\
\hline $\mathrm{Ni}+\mathrm{Cr}$ Homens & $8(2,0)$ & $10(2,0)$ & $17(5,2)$ & $12(3,0)$ & $47(3,0)$ & - \\
\hline $\mathrm{Ni}+\mathrm{Cr}$ Mulheres & $41(4,9)$ & $20(2,0)$ & $33(4,6)$ & $36(3,3)$ & $130(3,6)$ & - \\
\hline $\mathrm{Ni}+\mathrm{Co}+\mathrm{Cr}$ Total & $26(2,1)$ & $25(1,7)$ & $37(3,6)$ & $32(2,1)$ & $120(2,3)$ & - \\
\hline $\mathrm{Ni}+\mathrm{Co}+\mathrm{Cr}$ Homens & $5(1,3)$ & $9(1,8)$ & $13(4,0)$ & $7(1,7)$ & $34(2,1)$ & - \\
\hline $\mathrm{Ni}+\mathrm{Co}+\mathrm{Cr}$ Mulheres & $21(2,5)$ & $16(1,6)$ & $24(3,4)$ & $25(2,3)$ & $86(2,4)$ & - \\
\hline $\mathrm{Cr}+\mathrm{Co}$ Total & $54(4,4)$ & $58(3,9)$ & $56(5,4)$ & $43(2,9)$ & $211(4,0)$ & - \\
\hline $\mathrm{Cr}+\mathrm{Co}$ Homens & $28(7,1)$ & $40(8,2)$ & $27(8,3)$ & $12(3)$ & $107(6,6)$ & - \\
\hline $\mathrm{Cr}+\mathrm{Co}$ Mulheres & $26(3,1)$ & $18(1,8)$ & $29(4,1)$ & $31(2,8)$ & $104(2,9)$ & - \\
\hline $\begin{array}{l}\text { DCA ocupacional relacionada } \\
\text { com os metais }\end{array}$ & $112(9,0)$ & $99(6,7)$ & $74(7,1)$ & $57(3,8)$ & $342(6,5)$ & $r=-0,445$ \\
\hline $\begin{array}{l}\text { DCA relacionada com o Cr } \\
\text { na Construção Civil }\end{array}$ & $45(3,6)$ & $48(3,3)$ & $22(2,1)$ & $8(0,5)$ & $123(2,3)$ & $r=-0,639$ \\
\hline $\begin{array}{l}\text { DCA relacionada com o } \mathrm{Cr} \\
\text { com uso calçado }\end{array}$ & $12(1,0)$ & $17(1,2)$ & $16(1,5)$ & $16(1,1)$ & $61(1,2)$ & - \\
\hline
\end{tabular}

anos foi responsável por $49 \%$ das mulheres sensibilizadas nos primeiros cinco anos e apenas $32,6 \%$ nos últimos cinco, representado um decréscimo significativo das muIheres jovens sensíveis a este metal $(p<0,001)$ (Fig. 3). As variações na sensibilização ao Co nestes 20 anos não apresentaram significância estatística. No caso do Cr, a sensibilização a este metal decresceu sobretudo nos homens $(r=-0,535$, Fig. 4a).

A reactividade aos metais no TE foi considerada relevante em $89,7 \%$ dos casos (Tabela 1 ), na maioria dos casos relacionada com dermatose prévia e pelo uso de bijutaria ou outros objectos metálicos em contacto com a pele. A reação foi considerada de causa predominantemente profissional em 342 indivíduos $(6,5 \%)$, percentagem que
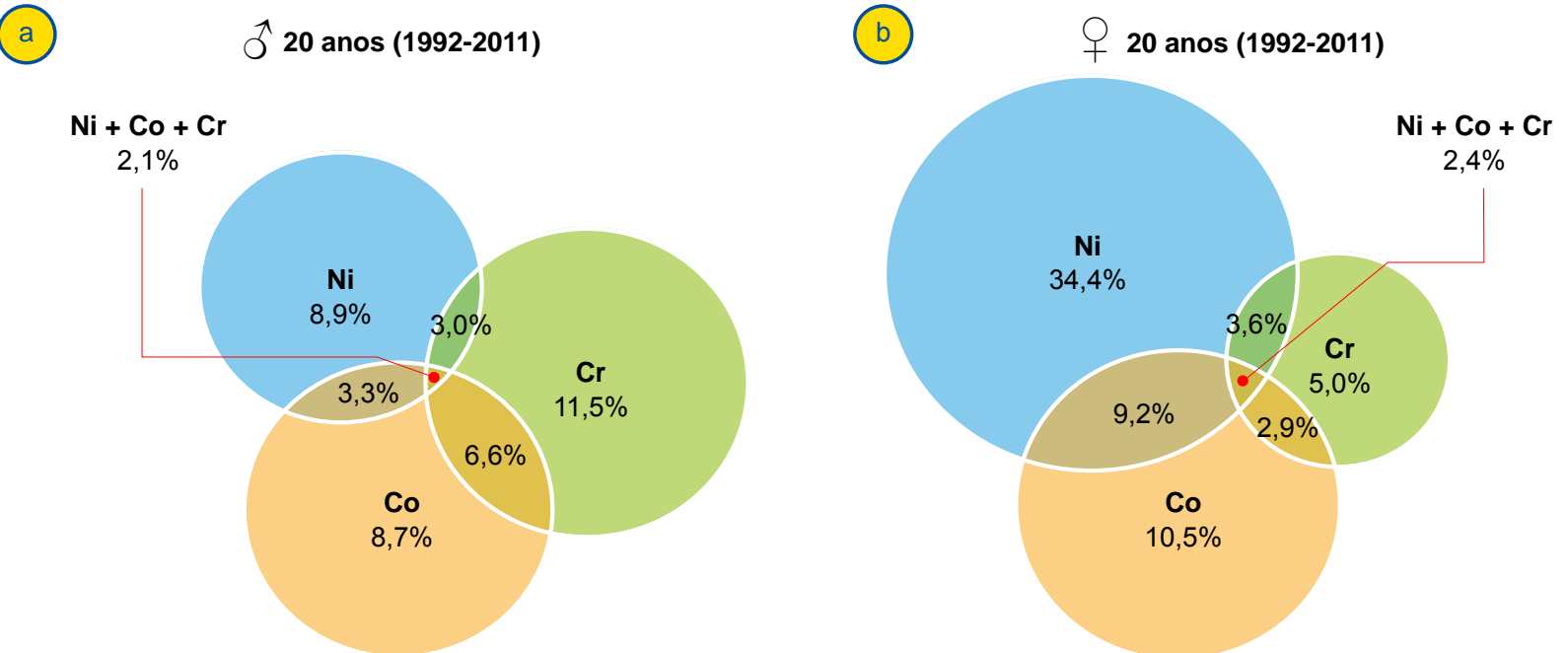

Figura 1 - Prevalência da co-sensibilização aos metais no sexo masculino (a) e feminino (b). 


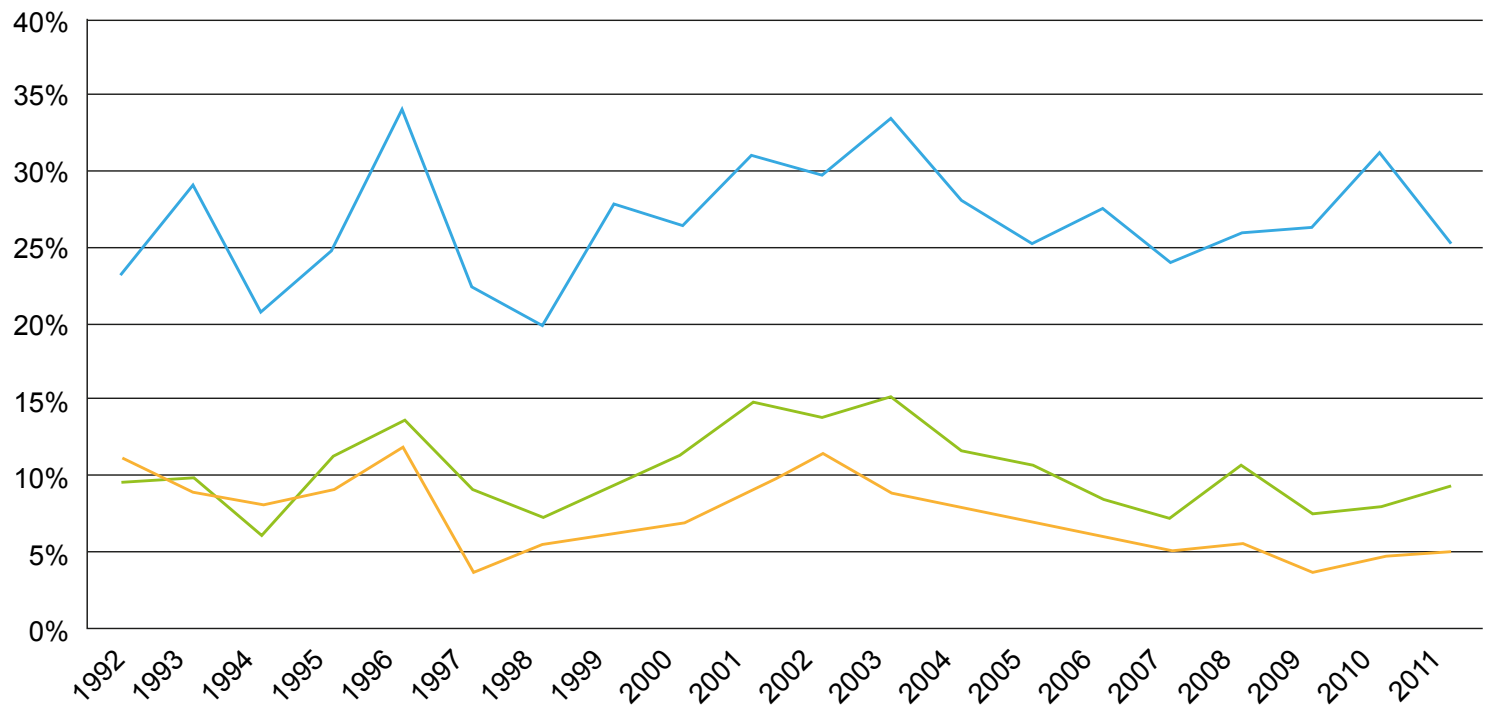

Níquel \% Cobalto $\% \quad$ Crómio \%

b \% Sensibilização aos metais - Homens

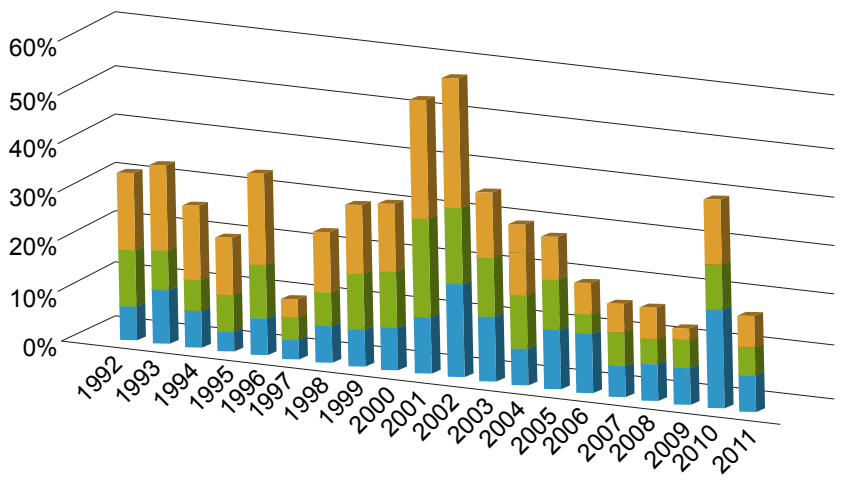

Níquel Cobalto Crómio

Figura 2 - (a) Padrões de variação da sensibilização aos metais nos últimos 20 anos. Variação temporal da sensibilização ao níquel, cobalto e crómio, no género masculino (b) e feminino (c).

ao longo dos vinte anos, tem vindo a diminuir de forma significativa $(r=-0,445 ; p=0,005$, Fig. $4 b)$. Esta redução é particularmente significativa no que se refere ao número de casos de DCA ao $\mathrm{Cr}$ nos trabalhadores da construção civil (3\% de todos os doentes testados em 1992 vs. $0,4 \%$ em 2011) (Fig. 5b). Pelo contrário, o número de casos de sensibilização ao $\mathrm{Cr}$ relacionado com o uso de calçado tem-se mantido estável ao longo dos anos (aprox. 1\%) (Fig. 5a).

\section{DISCUSSÃO}

Estudos do European Surveillance System on Contact
Allergy mostram que a sensibilização ao Ni é mais prevalente nos países na Europa do Sul. ${ }^{17}$ Em Espanha, a prevalência de alergia ao $\mathrm{Ni}$ ronda os $25 \%$, e é encarado como um problema de saúde pública. ${ }^{18,19}$ Os resultados do nosso estudo confirmam que os metais são uma importante causa de DCA na nossa população, sobretudo a alergia ao $\mathrm{Ni}$, afectando $26,5 \%$ dos doentes testados. A DCA causada pelo $\mathrm{Ni}$ pode ocorrer em contexto profissional (salões de cabeleireiro, restauração, metalúrgica, etc.), embora a exposição não-profissional predomine, como evidenciado no estudo de Rietschel et al em que apenas $6 \%$ dos 


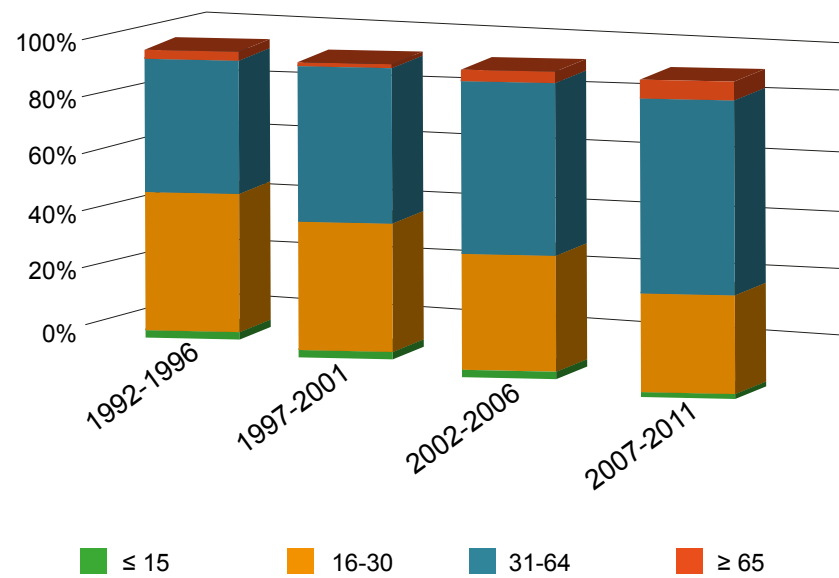

Figura 3 - Percentagem de mulheres sensibilizadas ao níquel, consoante a faixa etária. A faixa etária dos 16-30 anos representou uma percentagem decrescente das mulheres sensibilizadas a este metal nos últimos anos $(r=0,823, p<0,001)$.

\section{doentes alérgicos ao Ni tinham DCA ocupacional. ${ }^{20}$}

Causas multifactoriais (predisposição genética, exposição em oclusão ou de forma concomitante a irritantes cutâneos, dose, duração e frequência da exposição, assim como o local anatómico e área exposta) interferem na sensibilização ao Ni e, eventualmente, no grau de intensidade desta sensibilização. Assim, alguns indivíduos podem sofrer DCA ao Ni mesmo em doses abaixo do valor estabelecido pela Directiva comunitária, não sendo por isso possível estabelecer um valor-limite que garanta a evicção completa de DCA ao Ni em indivíduos sensibilizados (excepto a abolição completa do Ni dos objectos). ${ }^{15,21-23}$ No entanto, a aplicação destas medidas preventivas tem exercido efeitos positivos na sensibilização, reduzindo significativamente a percentagem da população sensibilizada ao $\mathrm{Ni}$, sobretudo

\section{Crómio}

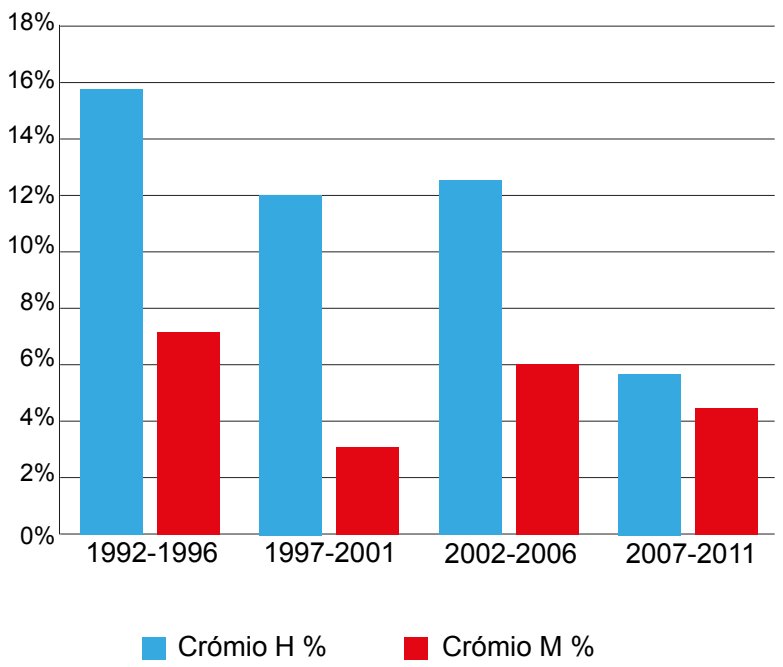

nos países nórdicos, pioneiros nesta iniciativa. ${ }^{14,15,24} \mathrm{Com}-$ provando o interesse destas medidas verifica-se que a sensibilização ao Ni na Dinamarca é significativamente inferior nas mulheres jovens cujas orelhas foram furadas depois da implementação destas medidas que regulamentam a quantidade de níquel libertada pelos adornos metálicos (odds ratio 1,20 vs 3,34$).{ }^{25}$

Pela análise dos dados do presente estudo infere-se que, para já, a Directiva do Níquel que foi transcrita para a legislação nacional apenas em 2005 poderá começar a ter algum efeito nas mulheres jovens. Contudo a sua implementação tardia, a incerteza quanto ao seu cumprimento integral e a exposição a objectos adquiridos antes da sua implementação ou em países não europeus em que não estão implementadas estas normas poderá justificar novos casos de sensibilização e a persistência do número de indivíduos sensibilizados, protelando o aparecimento dos efeitos benéficos destas medidas na sensibilização. $\mathrm{Na}$ faixa etária mais jovem ( $<16$ anos) em que o impacto da Directiva poderia ser mais evidente ao reduzir as novas sensibilizações, não verificámos diferenças significativas ao longo dos 20 anos, mas os números são baixos para ter expressão significativa. Como a faixa etária 16-30 anos é responsável por uma percentagem decrescente no total de mulheres sensibilizadas ao $\mathrm{Ni}$, poderemos inferir que se começa a sentir algum efeito benéfico das medidas comunitárias em relação a novas sensibilizações mas torna-se ainda assim necessário melhorar a implementação desta Directiva no nosso país.

A sensibilização ao cobalto acompanhou-se frequentemente de alergia ao $\mathrm{Ni}$ (sobretudo nas mulheres) ou ao crómio (nos homens). Num estudo retrospectivo, Thyssen et al mostraram que a alergia ao $\mathrm{Ni}$ tem vindo a diminuir nas mulheres jovens, enquanto a alergia ao Co se tem mantido
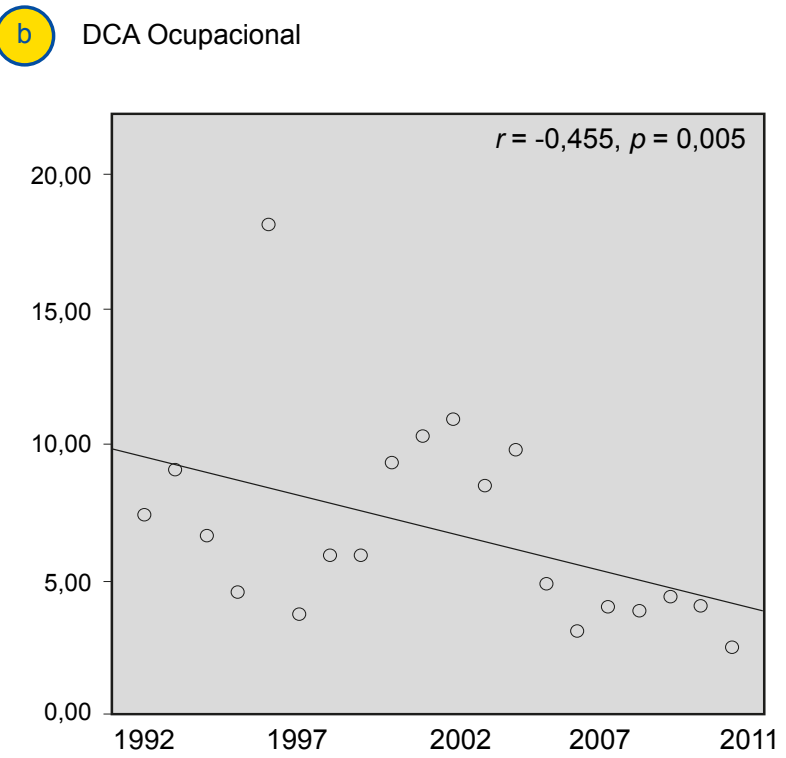

Figura 4 - (a) Diminuição do número de casos de alergia ao crómio, ao longo dos anos ( $r=-0,586, p=0,007)$, sobretudo no sexo masculino (azul no gráfico) $(r=-0,535, p=0,015)$. (b) Diminuição dos casos de DCA ocupacional relacionada com os metais $(r=-0,445$, $p=0,005)$. 


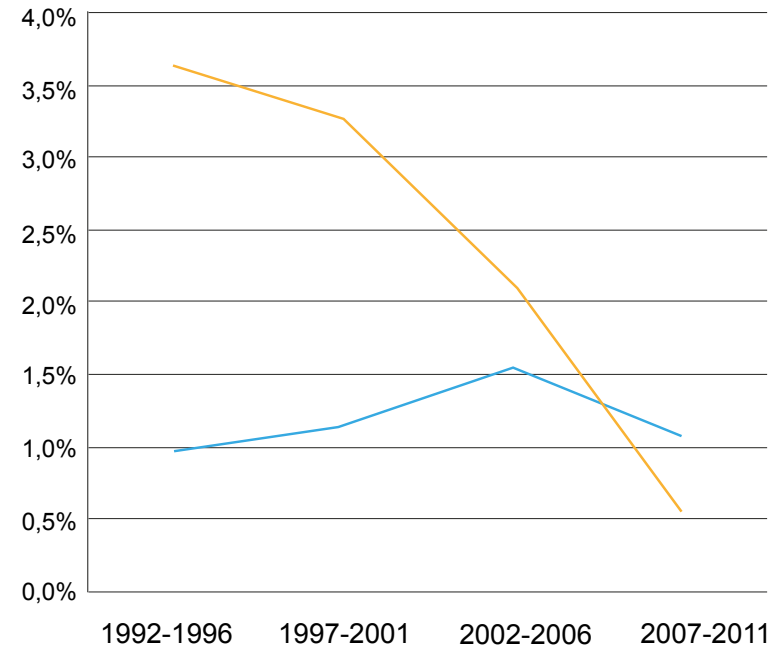

Construção Civil

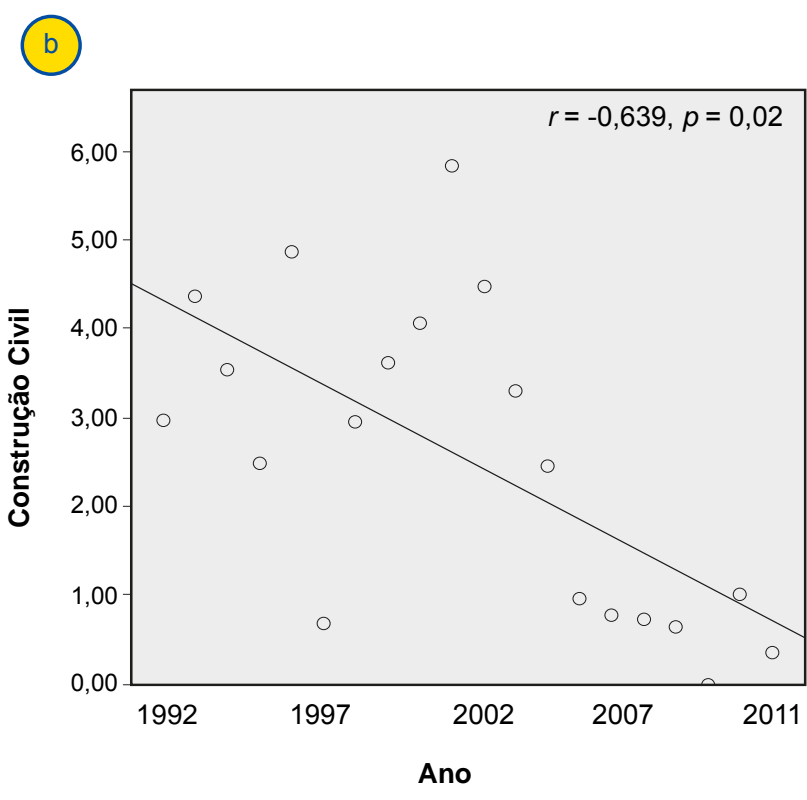

Figura 5 - (a) Relação entre DCA relacionada com o crómio nos trabalhadores da construção civil (linha laranja) vs DCA relacionada com o uso de calçado (linha azul). (b) Tendência decrescente do número de casos de alergia ao crómio entre os trabalhadores da construção civil $(r=-0,639, p=0,02)$.

estável. Sugerem que a provável substituição do Ni pelo Co p.e. na bijuteria poderá ser responsável por esta tendência, embora a libertação de Co testada nestes produtos tenha sido mínima. ${ }^{26,27}$

Nos últimos 20 anos, o número de casos de DCA ocupacional relacionada com os metais, na nossa população, tem vindo a diminuir de forma estatisticamente significativa. Isto poderá ser explicado, em parte, pela melhoria das condições de higiene-segurança no trabalho (em fábricas, indústria, construção civil, salões de cabeleireiro), com menor exposição aos alérgenos, mas também pela Directiva comunitária relacionada com o cimento.

Apesar de a DCA ocupacional nos trabalhadores da construção civil poder ser causada por múltiplos factores (metais, resinas epóxi, borrachas, etc.), dificultando a avaliação desta dermatose, o cimento é o produto mais importante neste contexto. A adição de sulfato ferroso como agente redutor permite transformar o $\mathrm{Cr}$ hexavelente na sua forma trivalente, insolúvel e com menor potencial sensibilizante. Este procedimento obrigatório na Dinamarca, desde 1983, traduziu-se numa diminuição drástica da prevalência de alergia ao $\mathrm{Cr}$ nesse contexto, embora com aumento dos casos relacionados com o uso de produtos de cabedal. ${ }^{28,29}$ No nosso estudo verificamos uma diminuição significativa dos casos de DCA causado pelo $\mathrm{Cr}$ nos trabalhadores da construção civil, apontando os efeitos positivos desta Directiva, transcrita para a legislação nacional em 2005.

Por outro lado, o $\mathrm{Cr}$ é frequentemente utilizado na indústria de curtimento da pele de animal, com o objectivo de aumentar a estabilidade, resistência e durabilidade desse material, para utilização por exemplo no calçado, onde causa frequentemente DCA. De modo geral, considera-se que existe risco de sensibilização e DCA quando a libertação de $\mathrm{Cr}$ VI dos materiais é superior a 3 ppm. ${ }^{30}$ No en- tanto, sob condições especiais (humidade, $\mathrm{pH}$, dermatoses pre-existentes, etc.) valores inferiores, a partir de $1 \mathrm{ppm}$, podem causar sensibilização. Os métodos analíticos disponíveis apenas detectam a libertação de $\mathrm{Cr}$ acima de 3 ppm ( $3 \mathrm{mg} / \mathrm{Kg}$ ). Um estudo recente Dinamarquês analisou o teor em $\mathrm{Cr}$ em 60 pares de calçado de pele comprados nesse país, embora alguns importados, inclusive de Portugal. ${ }^{30}$ Foram detectados valores elevados de $\mathrm{Cr}$ hexavalente, a maioria entre 3-6 ppm, mas alguns com valores atingindo 63 ppm. Esses resultados foram apresentados à European Chemicals Agency e, à data de elaboração deste artigo, a situação está a ser avaliada no sentido de implementar medidas no território da União Europeia também em relação ao $\mathrm{Cr}$ nos sapatos e outros produtos de couro para usar em contacto com a pele. Essas medidas serão baseadas nas restrições actualmente em vigor na Alemanha (desde Agosto de 2010), que consistem na proibição de venda de produtos de cabedal que permanecem em contacto prolongado com a pele (calçado, luvas, vestuário, malas) cujo teor de $\mathrm{Cr} \mathrm{VI}$ exceda concentrações de $3 \mathrm{mg} / \mathrm{Kg}$. Na nossa análise, o número de casos de alergia ao $\mathrm{Cr}$ relacionados com o uso de calçado não sofreu alterações significativas ao longo dos anos, confirmando a ausência de medidas regulamentares do teor de $\mathrm{Cr}$ no calçado também em Portugal.

O nosso estudo tem algumas limitações, inerentes à sua natureza retrospectiva e possível viés na selecção da amostra (doentes predominantemente com suspeita de dermite de contacto alérgica), podendo não ser representativa da população geral. No entanto, este estudo poderá servir de ponto de partida para futuras investigações, clarificando esta questão.

A regulamentação da implementação de medidas interventivas relacionadas quer com o fabrico e comércio 
de adornos (bijuteria/joalharia, relógios, etc.) quer a nível profissional (construção civil, fábricas têxteis, etc.) permitirá uma melhor protecção da população da alergia aos metais.

\section{AGRADECIMENTOS}

Os autores agradecem a Fátima Ribeiro toda a dedicação na preparação das provas de contacto e organização contínua dos ficheiros da Unidade de Alergologia Cutânea

\section{REFERÊNCIAS}

1. Nielsen $\mathrm{NH}$, Menné T. Nickel sensitisation and ear-piercing in an unselected Danish population. Glostrup Allergy Study. Contact Dermatitis. 1993;29:16-21.

2. Larsson-Stymne B, Widstrom L. Ear-piercing-a cause of nickel allergy in schoolgirls? Contact Dermatitis. 1985;13:289-93.

3. Wahlberg JE, Elsner P, Kanerva L, MaibachHI. Management of positive patch test reactions. New York: Springer; 2003.

4. Lidén C. Nickel in jewellery and associated products. Contact Dermatitis. 1992;26:73-5.

5. Vilaplana J, Romaguera C, Grimalt F, Cornellana F. New trends in the use of metals in jewellery. Contact Dermatitis. 1991;25:145-8.

6. Thyssen JP, Menné T, Johansen JD. Identification of metallic items that caused nickel dermatitis in Danish patients. Contact Dermatitis. 2010;63:151-6.

7. Aberer W, Kranke $B$. The new EURO releases nickel and elicits contact eczema. Br J Dermatol. 2002;146:155-6.

8. Nestle F O, Speidel H, Speidel MO. Metallurgy - high nickel release from 1-and 2-euro coins. Nature. 2002;419:132.

9. Yoshihisa $Y$, Shimizu T. Metal allergy and systemic contact dermatitis: an overview. Dermatol Res Pract. 2012;2012:749561.

10. Decreto-Lei $n^{\circ} 256 / 2000$ de 17 de Outubro. Diário da República n 240-I Série A. Lisboa: Ministério da Economia; 2000.

11. Decreto-Lei $n^{\circ} 222 / 2005$ de 27 de Dezembro. Diário da República ${ }^{\circ}$ 247-I Série A. Lisboa: Ministério da Economia e da Inovação; 2005.

12. Decreto-Lei $n^{\circ} 72 / 2005$ de 18 de Março de 2005. Diário da República ${ }^{\circ}$ 55-I Série A. Lisboa: Ministério das Actividades Económicas e do Trabalho; 2005.

13. Menné T, Brandup F, Thestrup-Pedersen K, Veien NK, Andersen JR, Yding $F$, et al. Patch test reactivity to nickel alloys. Contact Dermatitis. 1987;16:255-9.

14. Thyssen JP, Uter W, McFadden J, Menné T, Spiewak R, Vigan M, et al. The EU Nickel Directive revisited - future steps towards better protection against nickel allergy. Contact Dermatitis. 2011;64:121-5.

15. Thyssen JP. Nickel and cobalt allergy before and after nickel regulation - evaluation of a public health intervention. Contact Dermatitis. 2011;65:S1-68.

16. Wilkinson DS, Fregert S, Magnusson B, Bandmann HJ, Calnan CD, Cronin E, et al. Terminology of contact dermatitis. Acta Derm Venereol. 1970;50:287-92.

17. Uter W, Rämsch C, Aberer W, Ayala F, Balato A, Beliauskiene A, et al. The European baseline series in 10 European Countries, 2005/2006 results of the European Surveillance System on Contact Allergies (ESSCA). Contact Dermatitis. 2009;61:31-8. e a Margarida Marques do Serviço de Informática pela ajuda no tratamento estatístico.

\section{CONFLITO DE INTERESSES}

Sem conflito de interesses.

\section{FONTES DE FINANCIAMENTO}

Não existiram fontes de financiamento.

18. García-Gavín J, Armario-Hita JC, Fernández-Redondo V, FernándezVozmediano JM, Sánchez-Pérez J, Silvestre JF, et al. Nickel allergy in Spain needs active intervention. Contact Dermatitis. 2011;164:289-91.

19. Aguilar-Bernier M, Bernal-Ruiz Al, Rivas-Ruiz F, Fernández-Morano MT, de Troya-Martín M. Contact sensitization to allergens in the Spanish standard series at Hospital Costa del Sol in Marbella, Spain: a retrospective study (2005-2010). Actas Dermosifiliogr. 2012;103:223-8.

20. Rietschel RL, Mathias CGT, Fowler JF, Pratt M, Taylor JS, Sherertz EF, et al. Relationship of occupation to contact dermatitis: evaluation in patients tested from 1998 to 2000. Am J Contact Dermat. 2002;13:170-5.

21. Gawkrodger DJ. Nickel dermatitis: how much nickel is safe? Contact Dermatitis. 1996;35:267-71.

22. Fischer LA, Menne T, Johansen JD. Experimental nickel elicitation thresholds - a review focusing on occluded nickel exposure. Contact Dermatitis. 2005; 52:57-64.

23. Schram SE, Warshaw EM. Genetics of nickel allergic contact dermatitis. Dermatitis. 2007;18:125-33.

24. Thyssen JP, Linneberg A, Menné T, Nielsen NH, Johansen JD. The association between hand eczema and nickel allergy has weakened among young women in the general population following the Danish nickel regulation: results from two cross-sectional studies. Contact Dermatitis. 2009;61:342-8.

25. Thyssen JP, Johansen JD, Menné T, Nielsen NH, Linneberg A. Nickel allergy in Danish women before and after nickel regulation. N Engl JMed. 2009;360:2259-60.

26. Thyssen JP, Johansen JD, Carlsen BC, Menné T. Prevalence of nickel and cobalt allergy among female patients with dermatitis before and after Danish government regulation: a 23-year retrospective study. J Am Acad Dermatol. 2009;61:799-805.

27. Thyssen JP, Jellesen MS, Menné T, Lidén C, Julander A, Møller P, et al. Cobalt release from inexpensive jewellery: has the use of cobalt replaced nickel following regulatory intervention? Contact Dermatitis. 2010;63:70-6.

28. Zachariae $\mathrm{CO}$, Agner T, Menné T. Chromium allergy in consecutive patients in a country where ferrous sulfate has been added tocement since 1981. Contact Dermatitis. 1996;35:83-5.

29. Thyssen JP, Jensen P, Carlsen BC, Engkilde K, Menné T, Johansen JD. The prevalence of chromium allergy in Denmark is currently increasing as a result of leather exposure. Br J Dermatol. 2009;161:1288-93.

30. Thyssen JP, Strandesen M, Poulsen PB, Menné T, Johansen JD. Chromium in leather footwear - risk assessment of chromium allergy and dermatitis. Contact Dermatitis. 2012;66:279-85. 


\section{Alergia de Contacto a Metais num Período de 20 Anos no Centro de Portugal: Implicações das Directivas Europeias Acta Med Port 2014:27:295-303}

Publicado pela Acta Médica Portuguesa, a Revista Científica da Ordem dos Médicos

Av. Almirante Gago Coutinho, 151

1749-084 Lisboa, Portugal.

Tel: +351218428215

E-mail: submissao@actamedicaportuguesa.com

www.actamedicaportuguesa.com

ISSN:0870-399X | e-ISSN: 1646-0758

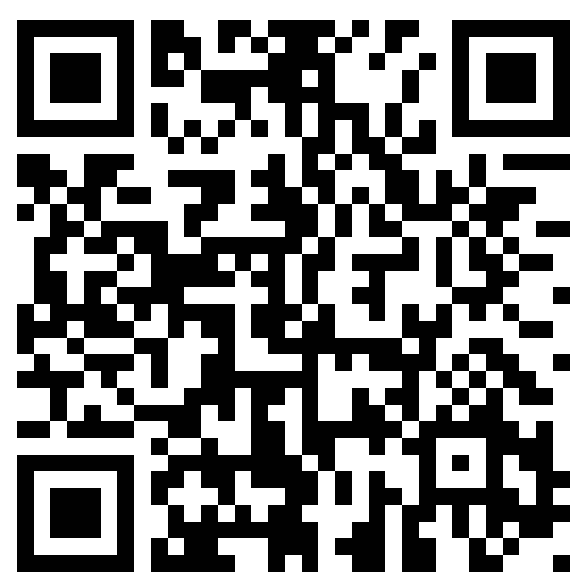

